\title{
Polarization-induced distortion in stimulated Brillouin scattering slow-light systems
}

\author{
Avi Zadok, ${ }^{1, *}$ Sanghoon Chin, ${ }^{2}$ Luc Thévenaz, ${ }^{2}$ Elad Zilka, ${ }^{3}$ Avishay Eyal, ${ }^{3}$ and Moshe Tur $^{3}$ \\ ${ }^{1}$ Department of Applied Physics, MC 128-95, California Institute of Technology, Pasadena, California 91125, USA \\ ${ }^{2}$ Ecole Polytechnique Fédérale de Lausanne, Institute of Electrical Engineering, STI-GR-SCI Station 11, 1015 \\ Lausanne, Switzerland \\ ${ }^{3}$ School of Electrical Engineering, Faculty of Engineering, Tel-Aviv University, Tel-Aviv 69978, Israel \\ *Corresponding author: avizadok@Caltech.edu
}

Received May 26, 2009; revised July 16, 2009; accepted July 23, 2009;

posted July 29, 2009 (Doc. ID 111932); published August 14, 2009

The vector analysis of stimulated Brillouin scattering amplification in birefringent fibers is extended to include signal pulses. The analysis finds that the different slow-light delays experienced by the states of polarization corresponding to maximum and minimum gain may result in severe pulse distortion. Thus, a generally polarized pulse, experiencing only a moderate gain, can become broader than a pulse aligned for maximum gain and delay. The effect is demonstrated in both numerical simulations and experiments. (C) 2009 Optical Society of America

OCIS codes: $290.5900,190.4370$.

Stimulated Brillouin scattering (SBS) is a nonlinear interaction, coupling a relatively strong optical pump wave with a counterpropagating, typically weaker signal wave, through a mediating, longitudinal acoustic wave [1]. When phase-matching requirements are met, SBS leads to an exponential amplification of signal waves. The SBS amplification is accompanied by frequency-dependent phase delays [1], which give rise to an effective added group delay of amplified signal pulses. SBS has become a favored underlying mechanism in many such variable groupdelay setups, often referred to as "slow light," for its low threshold power, robustness, and simplicity of operation $[2,3]$.

As SBS is driven by interference between the pump and the signal waves, the signal gain is known to be strongly and inherently dependent on polarization [4]. In recent work, a vector formalism was provided to describe SBS amplification in the presence of arbitrary fiber birefringence [5]. The analysis showed that in the undepleted pump regime, the SBSamplifying fiber is equivalent to a polarizationdependent gain medium. The maximum and minimum gains in that medium are associated with a pair of orthogonal signal states of polarization (SOPs), which are determined by the pump input SOP and the birefringence properties of the fiber [5]. Most previous work related to SBS polarization attributes was restricted, however, to CW signals [4-6]. The work in [7], although dedicated to signal pulses, did not examine the pulse distortion.

In SBS slow-light setups, pulse distortion due to the limited bandwidth and the dispersion associated with the scalar frequency dependence of SBS has been thoroughly documented [8]. In this Letter, we show that SBS-related polarization-induced distortion is yet another mechanism responsible for pulse broadening in slow-light setups. Basically, a signal pulse with its SOP aligned for maximum amplification undergoes a delay much longer than that expe- rienced by a pulse whose SOP is adjusted for minimum gain. Thus, it is shown below that this SBSrelated polarization induced distortion is analogous to that of linear birefringence, where the orthogonal SOPs of maximum and minimum gain have a role similar to that of the principal axes in linear birefringence-induced polarization mode dispersion (PMD) [9].

We start with the propagation equation for a single Fourier component of the signal Jones vector, $\vec{E}_{\text {sig }}(z, \Delta \omega)$, subject to both SBS amplification and birefringence, expressed by [5]

$$
\begin{aligned}
\frac{\mathrm{d} \vec{E}_{\text {sig }}(z, \Delta \omega)}{\mathrm{d} z}= & {\left[\frac{\mathrm{d} \mathbf{T}(z)}{\mathrm{d} z} \mathbf{T}^{\dagger}(z)+\frac{\gamma(\Delta \omega)}{2} \vec{E}_{\text {pump }}(z) \vec{E}_{\text {pump }}^{\dagger}(z)\right] } \\
& \times \vec{E}_{\text {sig }}(z, \Delta \omega) .
\end{aligned}
$$

Here $z$ denotes position along a fiber of length $L, \Delta \omega$ is the frequency detuning of the particular wave component from the frequency of maximum SBS amplification, and $\vec{E}_{\text {pump }}(z)$ is the Jones vector of an undepleted, monochromatic pump wave. The signal wave enters the fiber at $z=0$. The unitary $\mathbf{T}(z)$ represents the Jones matrix of linear propagation from the signal input up to point $z$. The SBS gain coefficient $\gamma(\Delta \omega)$ is of Lorenzian shape: $\gamma(\Delta \omega)=\gamma_{0} /(1$ $\left.+j 2 \cdot \Delta \omega / \Gamma_{B}\right)$, where $\gamma_{0}\left[(\mathrm{~W} \mathrm{~m})^{-1}\right]$ denotes the peak gain coefficient of SBS and $\Gamma_{B} / 2 \pi \approx 30 \mathrm{MHz}$ is the SBS linewidth [1]. The energy-conserving term $[\mathrm{d} \mathbf{T}(z) / \mathrm{d} z] \mathbf{T}^{\dagger}(z)$, which describes the local birefringence, is virtually frequency independent within the narrow $\Gamma_{B}$. This frequency independence would hold for broadened SBS processes, provided that linear PMD was negligible within the amplification bandwidth. Since Eq. (1) is linear, we may examine the propagation of signal pulses via Fourier component analysis. In a previous study [5], we obtained the following expression for a single-frequency signal Jones vector at the fiber output: 


$$
\begin{aligned}
\vec{E}_{\mathrm{sig}}(L, \Delta \omega)= & \mathbf{U}(\Delta \omega) \cdot\left[\begin{array}{cc}
G_{\max }(\Delta \omega) & 0 \\
0 & G_{\min }(\Delta \omega)
\end{array}\right] \\
& \cdot \mathbf{V}^{\dagger}(\Delta \omega) \cdot \vec{E}_{\mathrm{sig}}(0, \Delta \omega),
\end{aligned}
$$

where $\mathbf{U}, \mathbf{V}$ are unitary matrices and $G_{\max }, G_{\min }$ are the complex scalar amplitude transfer functions for maximum and minimum SBS gain, respectively. $G_{\max }, G_{\min }$ correspond to signal input unit vectors $\hat{e}_{\mathrm{sig}}^{\operatorname{in}_{\mathrm{ig}} \max }(\Delta \omega)=\mathbf{V} \cdot\left[\begin{array}{ll}1 & 0\end{array}\right]^{T}$ and $\hat{e}_{\mathrm{sig}}^{\mathrm{in} \min }(\Delta \omega)=\mathbf{V} \cdot\left[\begin{array}{ll}0 & 1\end{array}\right]^{T}$, respectively, and to signal output unit vectors

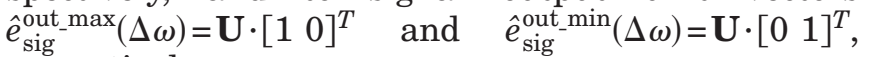
respectively.

In standard, birefringent fibers, $\hat{e}_{\mathrm{sig}}^{\mathrm{in} \max }(\Delta \omega)$ is closely aligned with the complex conjugate of $\vec{E}_{\text {pump }}(0)$, provided that $\gamma(\Delta \omega)\left|\vec{E}_{\text {pump }}\right|^{2}<2 \pi / L_{B}$, where $L_{B}$ is the mean beat length in the fiber [5]. The input $\operatorname{SOPs} \hat{e}_{\mathrm{sig}}^{\mathrm{in} \max }(\Delta \omega)$ and $\hat{e}_{\mathrm{sig}}^{\mathrm{in}_{\mathrm{i}} \min }(\Delta \omega)$ are therefore nearly frequency independent, even though $G_{\max }(\Delta \omega)$, $G_{\min }(\Delta \omega)$ vary exponentially with frequency through $\gamma(\Delta \omega)$. This frequency invariance is illustrated in the simulations results of Fig. 1, which show the unit three-element Stokes space representation $\hat{s}_{\mathrm{sig}}^{\mathrm{in}} \min (\Delta \omega)$ of $\hat{e}_{\mathrm{sig}}^{\mathrm{in} \min }(\Delta \omega)$. The inner product $p(\Delta \omega)$ $\equiv \hat{s}_{\mathrm{sig}}^{\mathrm{in} \min }(\Delta \omega)^{\dagger} \cdot \hat{s}_{\mathrm{sig}}^{\mathrm{in} \min }(0)$, in the particular example, always exceeded 0.94. $p(\Delta \omega)>0.95$ was also measured in an auxiliary experiment, by using the technique described in [5], for both a 2250-m-long fiber (pump power of 20-35 $\mathrm{mW}$ ) and a 415-m-long fiber $(150 \mathrm{~mW})$.

Based on the above, it is possible to replace Eq. (2) with a pair of decoupled, scalar SBS amplification equations, one associated with the frequency domain transfer function $G_{\max }(\Delta \omega)$ and the input SOP $\hat{e}_{\mathrm{sig}}^{\mathrm{in} \max }(0)$, and the other with $G_{\min }(\Delta \omega)$ and $\hat{e}_{\mathrm{sig}}^{\mathrm{in} \min }(0)$. It is convenient to examine the propagation of an arbitrarily polarized input signal pulse by using decomposition in the basis of $\hat{e}_{\mathrm{sig}}^{\mathrm{in} \max }(0)$ and $\hat{e}_{\mathrm{sig}}^{\mathrm{in} \min }(0)$. Since typically $G_{\max } \gg G_{\min }$, we expect that the SBSinduced delay of a pulse aligned with $\hat{e}_{\mathrm{sig}}^{\mathrm{in} \max }(0)$ will be longer than that of a pulse aligned with $\hat{e}_{\mathrm{sig}}^{\mathrm{in} \min }(0)$. These two SOPs, therefore, take up a role similar to that of the principal axes of linear birefringenceinduced PMD, on top of representing polarization de-

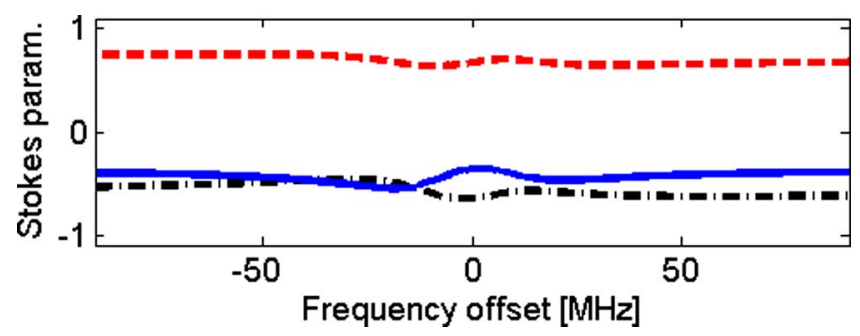

Fig. 1. (Color online) Calculated Stokes parameters $S_{1}$ (dashed), $S_{2}$ (dashed-dotted) and $S_{3}$ (solid) of the input signal that leads to the minimum SBS gain, as a function of detuning $\Delta \omega$ from the frequency of maximum SBS amplification, for a particular fiber realization. The simulation parameters were $L=140 \mathrm{~m}, L_{B}=40 \mathrm{~m}, L_{c}=10 \mathrm{~m},\left|\vec{E}_{\text {pump }}\right|^{2}$ $=560 \mathrm{~mW}, \gamma_{0}=0.16(\mathrm{~W} \mathrm{~m})^{-1}$, and $\Gamma_{B} / 2 \pi=30 \mathrm{MHz}$. pendent gain axes. The large difference in gain is also responsible for the previously reported pulling of the output SOP of CW signals toward that of $\hat{e}_{\text {sig }}^{\text {outmax }}(0)$ [5].

The predicted SBS-induced distortion was first verified by using numerical simulations of Eq. (1). Figure 2 shows examples of calculated, normalized signal output power as a function of time $t$, obtained for a signal input Gaussian pulse with a FWHM of $17 \mathrm{~ns}$ and for several signal input SOPs. The simulation parameters were $\left|\vec{E}_{\text {pump }}\right|^{2}=560 \mathrm{~mW}, L=140 \mathrm{~m}$, and $L_{B}=40 \mathrm{~m}$ with a birefringence correlation length $L_{c}$ of $10 \mathrm{~m}$ [10]. We designate output power of pulses aligned with $\hat{e}_{\mathrm{sig}}^{\mathrm{in} \max }(0)$ and $\hat{e}_{\mathrm{sig}}^{\mathrm{in} \min }(0)$ as $P_{\mathrm{sig}}^{\text {out } \max }(t)$ and $P_{\text {sig }}^{\text {outmin }}(t)$, respectively. The SBS gain, group delay, and output FWHM of $P_{\text {sig }}^{\text {outmax }}(t)$ were $33 \mathrm{~dB}, 38 \mathrm{~ns}$, and $54.5 \mathrm{~ns}$, respectively. The corresponding values for $P_{\text {sig }}^{\text {outmin }}(t)$ were $9 \mathrm{~dB}, 7 \mathrm{~ns}$, and $26 \mathrm{~ns}$. Dasheddotted curves in Fig. 2 correspond to intermediate in-

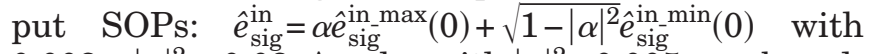
$0.002<|\alpha|^{2}<0.02$. A pulse with $|\alpha|^{2}=0.005$ was broadened to a FWHM of $74 \mathrm{~ns}$, even though its SBS gain was only $10.8 \mathrm{~dB}$ ! The near-minimum input polarizations shown in the figure represent a worst case scenario for the pulse broadening. Since $G_{\max } \gg G_{\min }$, the normalized output pulse shape would rapidly approach that of $P_{\text {sig }}^{\text {out max }}(t)$ when $|\alpha|^{2}$ is increased, as seen in the rightmost dashed-dotted curve $\left(|\alpha|^{2}\right.$ $=0.02$ ).

Figure 2 also shows the normalized output shape associated with the approximate output power $|\alpha|^{2} P_{\text {sig }}^{\text {outmax }}(t)+\left(1-|\alpha|^{2}\right) P_{\text {sig }}^{\text {outmin }}(t), \quad|\alpha|^{2}=0.002$. This first-order approximation is close to the exact solution, with the excessive pulse broadening well ac-

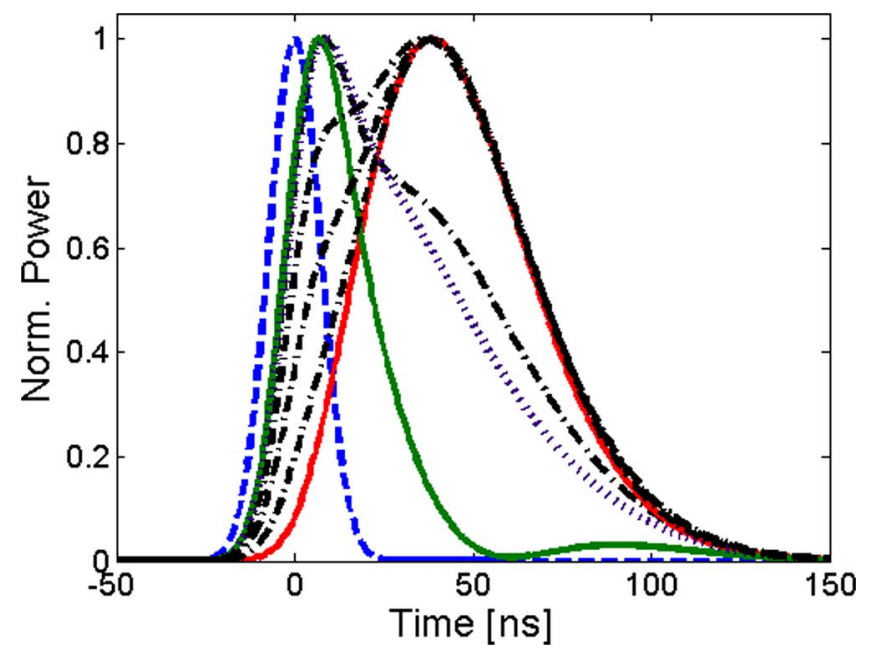

Fig. 2. (Color online) Calculated, normalized signal power as a function of time. Dashed blue curve, input Gaussian pulse (FWHM $17 \mathrm{~ns}$ ). Solid curves, output pulses with the input SOP aligned for minimum gain (left, green) and maximum gain (right, red). Black dashed-dotted curves, examples of output pulses with intermediate input SOP alignments. Dotted curve, approximate output pulse, corresponding to the nearest dashed-dotted curve, calculated by using a decomposition of the near-minimum input SOP in the basis of $\hat{e}_{\mathrm{sig}}^{\mathrm{in} \max }(0), \hat{e}_{\mathrm{sig}}^{\mathrm{in} \min }(0)$. The simulation parameters were the same as those of Fig. 1. 
counted for by a first-order representation of SBSinduced PMD. The two constituent components of the output pulse may be separated, to a good approximation, by a properly aligned output polarizer. The small deviations between the exact and the approximate solutions are due to the weak frequency dependence of $\hat{e}_{\mathrm{sig}}^{\text {in max }}, \hat{e}_{\mathrm{sig}}^{\mathrm{in}_{\mathrm{g}} \min }$ (see Fig. 1). The approximation may no longer hold at very high pump-power levels, which might lead to stronger frequency variations of the two vectors.

Excessive polarization-related pulse broadening was also observed experimentally. The measurement setup is shown in Fig. 3 [5]. Light from a distributed feedback laser diode (DFB-LD) was split by a directional coupler. One branch was amplified by an erbium-doped fiber amplifier (EDFA) and was launched into the fiber under test as an SBS pump wave at $z=L$. The other branch was double-sideband modulated at the Brillouin frequency shift of the fiber under test $(10.91 \mathrm{GHz})$, with the bias of the electrooptic modulator (EOM) adjusted to suppress the optical carrier frequency. The upper frequency sideband was discarded by a narrowband fiber Bragg grating (FBG), and the filtered lower frequency sideband was used as a Stokes wave signal. This signal was then modulated by Gaussian pulses, using a second EOM, and was launched into the fiber at $z=0$. A polarization controller (PC) was used to adjust the input signal SOP. The pump power, fiber length, and input pulse FWHM were $560 \mathrm{~mW}, 140 \mathrm{~m}$ and $17 \mathrm{~ns}$, as in the simulations.

Figure 4 shows measurements of the normalized output power for different input SOPs. The gain and FWHM of $P_{\text {sig }}^{\text {outmax }}(t)$ were $21 \mathrm{~dB}$ and $63 \mathrm{ns,}$, whereas those of $P_{\text {sig }}^{\text {outmin }}(t)$ were $6.7 \mathrm{~dB}$ and $30 \mathrm{~ns}$. Several pulses of intermediate SOP alignments, amplified by only $9.5-13 \mathrm{~dB}$, were broadened to an FWHM of 65-75 ns. The results demonstrate that SBS slow light implementations may introduce a polarizationrelated distortion, which is inherent to the vector nature of SBS. Polarization-induced distortion becomes negligible when the signal input is closely aligned with $\hat{e}_{\mathrm{sig}}^{\mathrm{in} \max }$, which is the preferable input SOP in most slow light setups. Nonetheless, our results show that an arbitrarily polarized signal pulse, subject to a comparatively moderate amplification, can become broader than a pulse aligned for maximum gain and

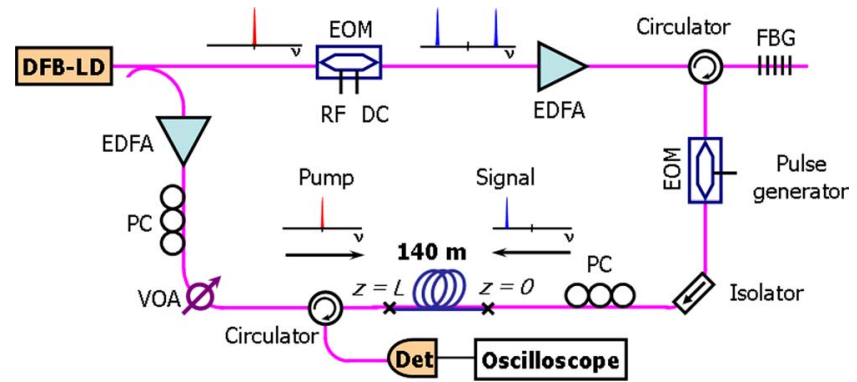

Fig. 3. (Color online) Experimental setup for observing SBS PMD. VOA, variable optical attenuator; Det, detector; $\mathrm{RF}$, radio frequency; $\mathrm{DC}$, direct current.

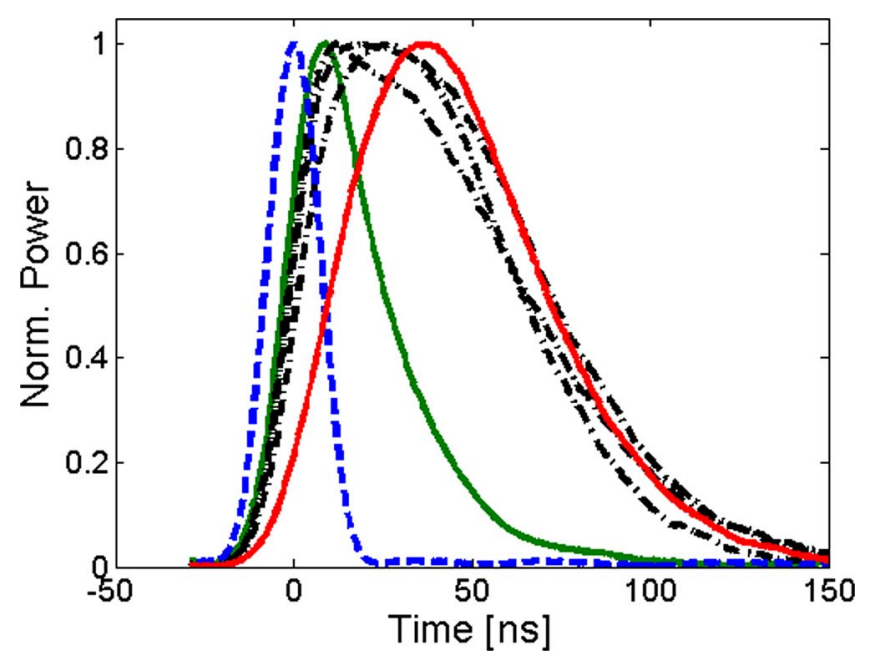

Fig. 4. (Color online) Measured, normalized signal power as a function of time. Dashed blue curve, input Gaussian pulse (FWHM $17 \mathrm{~ns}$ ). Solid curves, output pulses with the input SOP aligned for minimum gain (left, green) and maximum gain (right, red). Black dashed-dotted curves, examples of output pulses with intermediate input SOP alignments. Experimental conditions: $L=140 \mathrm{~m},\left|\vec{E}_{\text {pump }}\right|^{2}$ $=560 \mathrm{~mW}$.

delay. Unless polarization is stabilized, the width of the maximally delayed pulse does not necessarily set an upper bound on pulse broadening in SBS slow light delay setups.

We acknowledge support from the Swiss National Science Foundation (project 200020_121860), the European Community's 7th Framework Programme (GOSPEL project 219299), the European COST Action 299 "FIDES," and the Israeli Science Foundation (project 297/07). A. Zadok acknowledges a postdoctoral fellowship from the Center of Physics in Information (CPI), Caltech.

\section{References}

1. R. W. Boyd, Nonlinear Optics (Academic, 2003), Chap. 9, pp. 409-427.

2. L. Thévenaz, Nat. Photonics 2, 474 (2008).

3. G. M. Gehring, R. W. Boyd, A. L. Gaeta, D. J. Gauthier, and A. E. Willner, J. Lightwave Technol. 26, 3752 (2008).

4. M. O. van Deventer and A. J. Boot, J. Lightwave Technol. 12, 585 (1994).

5. A. Zadok, E. Zilka, A. Eyal, L. Thévenaz, and M. Tur, Opt. Express 16, 21692 (2008).

6. A. Galtarossa, L. Palmieri, M. Santaguistina, L. Schenato, and L. Ursini, IEEE Photon. Technol. Lett. 20, 1420 (2008).

7. D. R. Walker, M. Bashkanski, A. Gulian, F. K. Fatemi, and M. Steiner, J. Opt. Soc. Am. B 25, C61 (2008).

8. Z. Zhu, D. J. Gauthier, Y. Okawachi, J. E. Sharping, A. L. Gaeta, R. W. Boyd, and A. E. Willner, J. Opt. Soc. Am. B 22, 2378 (2005).

9. J. P. Gordon and H. Kogelnik, Proc. Natl. Acad. Sci. USA 97, 4541 (2000).

10. P. K. A. Wai and C. R. Menyuk, J. Lightwave Technol. 14, 148 (1996). 